\title{
Hand anlegen!
}

$\mathrm{D}$ er amerikanische Osteopath Steve Paulus staunte nicht schlecht, als er dem berühmten Stanley Schiowitz D.O. vorgestellt wurde, und dieser meinte, man sei sich zwar jetzt begegnet, aber es bestehe noch keine osteopathische Bruderschaft. Erst nach hartnäckigem Nachfragen lernte Steve, dass er nicht etwa eine wichtige Initiation verpasst habe, sondern dass sie sich gegenseitig noch nicht behandelt hätten. Dies wurde bei nächster sich bietender Gelegenheit nachgeholt.

Die Geschichte offenbart einen wichtigen Aspekt osteopathischen Miteinanders. Früher gehörte es zum medizinischen Ethos sich unter Kollegen zu behandeln - und das kostenlos. Das ist Vergangenheit. Das gegenseitige Hand anlegen erfährt aber im Bereich der Osteopathie offensichtlich eine Wiederbelebung. Dieser Umstand ist in vielerlei Hinsicht nützlich und angenehm. Leider zählt das gegenseitige Üben im Rahmen der Ausbildung oder in Kursen nicht zum gegenseitigen Behandeln, da dies meist unter Fragestellungen geschieht, die nichts mit den gesundheitlichen Problemen des momentanen „Opfers“ zu tun haben.

Gegenseitiges Behandeln hilft Brücken schlagen. Misstrauen Kollegen gegenüber, die an anderen Schulen ihre Ausbildung durchlaufen haben, schwindet, wenn man deren wissende, sehende und denkende Finger wahrnimmt. Aha, woanders wird offensichtlich nicht nur unterrichtet, weil man damit Geld verdienen will, sondern mit Qualitätsanspruch. An den Früchten erkennt man einander. Es gehört schon ein Vertrauensvorschuss dazu, sich unter die Hände eines anderen zu begeben, sich sozusagen auszuliefern. Wird die Behandlung den eigenen Erwartungen gerecht werden? Kommen harte Techniken zum Einsatz, obwohl man selber diese ablehnt?

Es ist unbestritten, dass, wer ganzheitlich heilend tätig ist, in sich selber möglichst gesund sein sollte, um nicht eigene Probleme auf die Mitmenschen zu übertragen. Wer ist jedoch stets völlig ausgeglichen in seiner Mitte? Außerdem steigt mit zunehmender Sensibilität für die Probleme anderer auch die Wahrnehmungsfähigkeit für Störungen im eigenen Körper. Eine ganz wichtige Position sollte die Pflege unseres Handwerkszeugs einnehmen: die unserer Hände. Jeder Handwerker achtet darauf, dass die Arbeitsmaterialien in einem optimalen Zustand sind. Tun wir das auch immer oder wiegen wir uns in dem Glauben, die Hände werden schon in Ordnung sein, weil wir nichts Gegenteiliges spüren?

Es gibt sicher genügend Argumente, sich in die Hände von Kolleginnen und Kollegen zu begeben, und sei es dass man einfach selber einmal wieder verspürt, wie Osteopathie wirkt, was eventuell Patienten erleiden, die eine Erstverschlimmerung durchmachen. Und so wird am Rand von Kursen, in der Mittagspause oder abends im Hotelzimmer immer wieder gegenseitig Hand angelegt. Ein schöner Brauch - keine Selbstverständlichkeit!

Eine besondere Art osteopathischer Bruder- oder eher Schwesternschaft wurde unserer Mitherausgeberin Marina Fuhrmann, D.O. M.R.O., zuteil. Die A.T. Still University in Kirksville, Ohio, verlieh ihr den Titel eines Doctor of Osteopathic Education, D.O.Ed. Sie nahm die Ehrung entgegen im Namen aller Osteopathen und werdenden Osteopathen in Deutschland und Europa. Das gesamte Team der DO gratuliert ihr herzlich. 